\title{
Controlling chaotic transients: Yorke's game of survival
}

\author{
Aguirre, Jacobo; D'ovidio, Francesco; Sanjuán, Miguel A. F.
}

Published in:

Physical Review E. Statistical, Nonlinear, and Soft Matter Physics

Link to article, DOI:

10.1103/PhysRevE.69.016203

Publication date:

2004

Document Version

Publisher's PDF, also known as Version of record

Link back to DTU Orbit

Citation (APA):

Aguirre, J., D'ovidio, F., \& Sanjuán, M. A. F. (2004). Controlling chaotic transients: Yorke's game of survival. Physical Review E. Statistical, Nonlinear, and Soft Matter Physics, 69(1), 016203.

https://doi.org/10.1103/PhysRevE.69.016203

\section{General rights}

Copyright and moral rights for the publications made accessible in the public portal are retained by the authors and/or other copyright owners and it is a condition of accessing publications that users recognise and abide by the legal requirements associated with these rights.

- Users may download and print one copy of any publication from the public portal for the purpose of private study or research.

- You may not further distribute the material or use it for any profit-making activity or commercial gain

- You may freely distribute the URL identifying the publication in the public portal

If you believe that this document breaches copyright please contact us providing details, and we will remove access to the work immediately and investigate your claim. 


\title{
Controlling chaotic transients: Yorke's game of survival
}

\author{
Jacobo Aguirre, ${ }^{1, *}$ Francesco d'Ovidio, ${ }^{2,3, \dagger}$ and Miguel A. F. Sanjuán ${ }^{1, \hbar}$ \\ ${ }^{1}$ Nonlinear Dynamics and Chaos Group, Departamento de Matemáticas y Física Aplicadas y Ciencias de la Naturaleza, \\ Universidad Rey Juan Carlos, Tulipán s/n, 28933 Móstoles, Madrid, Spain \\ ${ }^{2}$ Quantum Protein Group, Department of Physics, Technical University of Denmark, 2800 Lyngby, Denmark \\ ${ }^{3}$ Instituto Mediterráneo de Estudios Avanzados, IMEDEA (CSIC-UIB), Campus Universitat Illes Balears,
}

E-07071 Palma de Mallorca, Spain

(Received 2 September 2003; published 20 January 2004)

\begin{abstract}
We consider the tent map as the prototype of a chaotic system with escapes. We show analytically that a small, bounded, but carefully chosen perturbation added to the system can trap forever an orbit close to the chaotic saddle, even in presence of noise of larger, although bounded, amplitude. This problem is focused as a two-person, mathematical game between two players called "the protagonist" and "the adversary." The protagonist's goal is to survive. He can lose but cannot win; the best he can do is survive to play another round, struggling ad infinitum. In the absence of actions by either player, the dynamics diverge, leaving a relatively safe region, and we say the protagonist loses. What makes survival difficult is that the adversary is allowed stronger "actions" than the protagonist. What makes survival possible is (i) the background dynamics (the tent map here) are chaotic and (ii) the protagonist knows the action of the adversary in choosing his response and is permitted to choose the initial point $x_{0}$ of the game. We use the "slope 3" tent map in an example of this problem. We show that it is possible for the protagonist to survive.
\end{abstract}

DOI: 10.1103/PhysRevE.69.016203

PACS number(s): 05.45.Gg, 05.45.Pq

Transient chaos [1] is an interesting physical phenomenon which occurs in systems where trajectories bounce chaotically for a certain time in a bounded region until they reach a final state, usually nonchaotic. Varied manifestations of transient chaos are present in chaotic scattering [2], chaotic advection in fluid dynamics [3], species competition in ecology [4,5], or voltage collapse in electric power systems $[4,6]$, to cite just a few. From the point of view of nonlinear dynamics, the phenomenon of transient chaos is associated with the existence of a certain type of sets called chaotic saddles, also known as nonattracting chaotic invariant sets, formed by a bounded set of unstable periodic and aperiodic orbits, for which almost all trajectories diverge. Typical orbits in the system will approach the chaotic saddle following its stable manifold, spend some time bouncing in its vicinity, and then escape from it following its unstable manifold. Therefore, a compelling challenge might be to find a simple method to maintain an orbit in the neighborhood of the invariant set for all times, respecting the original dynamics of the system.

While for a linear system the perturbation needed to change its nature is of the same order as the dynamics of the motion, the extreme sensitivity to initial conditions makes control with very little perturbations a possible task. In this sense, diminishing the amplitude of control is an important goal in this field. Obviously, if the system is embedded in a noisy environment controlling orbits is even harder, and typically stronger amplitudes than in the noiseless case are needed. Since the seminal paper of Ott, Grebogi, and Yorke [7], the theory of chaos control in nonlinear dynamics has

\footnotetext{
*Email address: jaguirre@escet.urjc.es

†Email address: dovidio@imedea.uib.es

†Email address: msanjuan@escet.urjc.es
}

been thoroughly developed, both for Hamiltonian and dissipative systems. Most of the work has been focused on systems with chaotic attractors, both in noiseless and noisy environments [8], and comparatively less attention has been paid to the control of chaotic saddles. Nevertheless, examples of studies of transient chaos include theoretical works as well as applications for models of ecological, electrical, chemical, and laser systems [4,9-12]. The aim of these works is typically to find small perturbations that keep the system in a permanent chaotic regime. While such strategies have to deal essentially with the unstable dynamics of the saddle, in the present work we shall emphasize the destabilizing effect of strong noise. Thus our goal is to find a strategy able to trap the system close to the chaotic saddle indefinitely even in the presence of noise stronger than control.

Since Akiyama and Kaneko presented the "dynamical systems game theory" [13-15], there has been a growing interest for modeling increasingly more complex game strategies with concepts borrowed from nonlinear dynamics. In their work it is shown that game theory has resulted to be deeply related to several problems involving dynamical phenomena, and for many cases it is possible to switch from the point of view of game theory to that of nonlinear dynamics. In fact, the nature of these games can be described as a dynamical system. Our work points in this direction, and we face our problem as a mathematical game between two players called "the protagonist" and "the adversary," the protagonist's goal is to survive inside a bounded region, that is, the vicinity of the chaotic saddle. We describe an idea which we apply here to a very simple nonlinear dynamical system, but can be conveniently adapted for a wide variety of maps with a chaotic saddle, in which some kind of noise and control is present. In a system with attractors, the natural ten- 
dency of a particle is to reach one of these attractors, and therefore it is plausible for the protagonist to maintain itself close to one attractor even when the adversary is allowed slightly stronger actions. However, it is important to remark that without any external control, the probability of the protagonist to survive in the vicinity of a chaotic saddle is zero, even in the absence of noise, and this fact makes the survival of the protagonist a remarkable achievement.

The simplest form of this game involves a onedimensional map, the tent map, that is defined as $T(x)$ $=m(1-|x|)-1$. For cases of interest such as $m=3$, almost all initial points $x_{0}$ yield trajectories of $x_{n+1}=T\left(x_{n}\right)$ that go to $-\infty$ as $n \rightarrow \infty$; and in this case we say the protagonist does not survive. To survive he must act. The equation of the game is

$$
x_{n+1}=T\left(x_{n}\right)+u_{n+1}+r_{n+1},
$$

where the adversary chooses the perturbation $u_{n+1}$ (knowing $x_{n}$ and $T$ ) and the protagonist then chooses the "response" $r_{n+1}$ (knowing $u_{n+1}$ and $x_{n}$ and $T$ ). The perturbation $u_{n+1}$ might be chosen at random or using an effective strategy. In the long run there is little difference between these two if the protagonist can survive forever. The protagonist faces what appears to be an impossible task because we permit only $\left|u_{n}\right| \leqslant u_{0}$ and $\left|r_{n}\right| \leqslant r_{0}$, where $r_{0}$ and $u_{0}$ are specified with $r_{0}$ $<u_{0}$. If $r_{n}$ is viewed as the control and $u_{n}$ is viewed as some kind of noise (or interference), the usual requirement is that the control is stronger than the noise. However, the main goal of this paper is to show that in the context of transient chaos it is possible to control a noisy orbit, even in the case in which noise is stronger than control. The smaller bound on $r_{n}$ than on $u_{n}$ might lead us to call $r_{n}$ an "influence" rather than a "control" since the protagonist cannot control the details of the trajectory. For this problem, we let the "relatively safe" region be the interval $S=[-1,+1]$ and terminate the game if some $x_{n}$ is outside $S$. Certainly if $x_{n}$ is outside $S$, it is possible for the adversary to choose the sequence $u_{n}$ that causes the sequence $x_{n}$ to diverge, and there is a slightly larger interval depending on $u_{0}$ and $r_{0}$ such that if $x_{n}$ is outside that, the trajectory must diverge even if the adversary tries to help. To keep formulas simple, we state our results for $m=3$ though analogous results are available for all $m>2$. (If $m \leqslant 2$, there is a chaotic attractor and if $u_{0}$ is sufficiently small, survival is guaranteed even if the response size is 0 .) We begin with an example.

For $u_{0}=4 / 9$ and $r_{0}=2 / 9$, there exists a strategy guaranteeing survival. If $u_{0}>2 r_{0}$, then there is no strategy guaranteeing survival.

The best strategy for survival depends on $r_{0}$ as is made clear in the following theorem. There are different strategies for $r_{0} \geqslant 2 / 3$ and each integer $k$, where $r_{0}$ is in $\left[2 / 3^{k}, 2 / 3^{k-1}\right.$ ). Recall $m=3$.

Theorem. There is a strategy guaranteeing survival for a given $r_{0}$ and $u_{0}$ if and only if there is an integer $k \geqslant 1$ for which $2 / 3^{k} \leqslant r_{0}$ and $u_{0} \leqslant r_{0}+2 / 3^{k}$. (The cross-hatched part of Fig. 1 shows where there are strategies for survival.)

This type of problem is quite different from the standard control in which the goal is to drive the trajectory to a point.

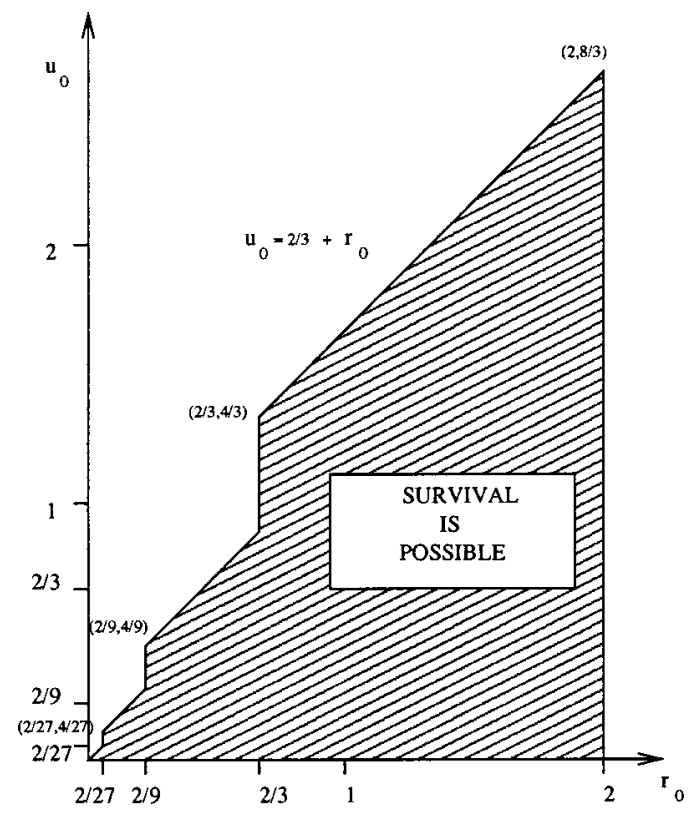

FIG. 1. Parameter region of survival. Survival is possible in the cross-hatched region if the protagonist chooses optimally. Above the cross-hatched region, the adversary can always win.

In controlling chaos [7,9], for example, if noise is present (i.e., $u_{n}$ chosen at random), the control $r_{n}$ must dominate $u_{n}$ so as to be able to drive the trajectory to a specified fixed point and keep it close to the fixed point. In the game of survival for the tent map, there are several "safety points" and $r_{0}$ must be large enough so that the protagonist can reach one of them, but the choice of which is really determined by what $u_{n}$ happens to be. The protagonist is bounced between these safety points in an order determined by the sequence of $u_{n}$.

The example. Before analyzing the theorem in detail, we examine the case mentioned above, $u_{0}=4 / 9$ and $r_{0}=2 / 9$ and show that the protagonist can survive. We designate four points as safety points, $z_{1}=-2 / 3-2 / 9, z_{2}=-2 / 3+2 / 9, z_{3}$ $=+2 / 3-2 / 9$, and $z_{4}=+2 / 3+2 / 9$. It is easy to check that $T\left(z_{i}\right)= \pm 2 / 3$ and $T( \pm 2 / 3)=0$. A graph of the tent map appears in Fig. 2 showing all these points, and Fig. 3 shows the evolution of an orbit in this situation. The protagonist's strategy must be to make sure every $x_{n}$ in Eq. (1) is a safe point if it is to guarantee that he can survive. In particular, the protagonist must choose $x_{0}$ equal to one of the safety points to make sure he succeeds (although in fact most points in $S=[-1,1]$ would also be valid as $x_{0}$ ). If $x_{n}$ is a safety point for any integer $n \geqslant 0$, then we show he can choose $r_{n+1}$ so that $x_{n+1}$ is a safety point, and so he survives another day. Since $x_{n}$ is a safety point, we may suppose, for example, $T\left(x_{n}\right)$ is $+2 / 3$. (The case $-2 / 3$ is virtually the same.) Then after $u_{n+1}$ is chosen, the point $T\left(x_{n}\right)+u_{n+1}$ must be in the interval $[2 / 3-4 / 9,2 / 3+4 / 9]$ and so is at most $2 / 9$ from either $z_{3}$ or $z_{4}$. Hence $r_{n+1}$ can be chosen with $\left|r_{n+1}\right| \leqslant r_{0}$ so that $x_{n+1}$ is a safety point. This case may be generalized by noting that this strategy works whenever $u_{0}-r_{0} \leqslant 2 / 9$.

This example illustrates why we call this problem a game of "survival" rather than of "control," since the protagonist 


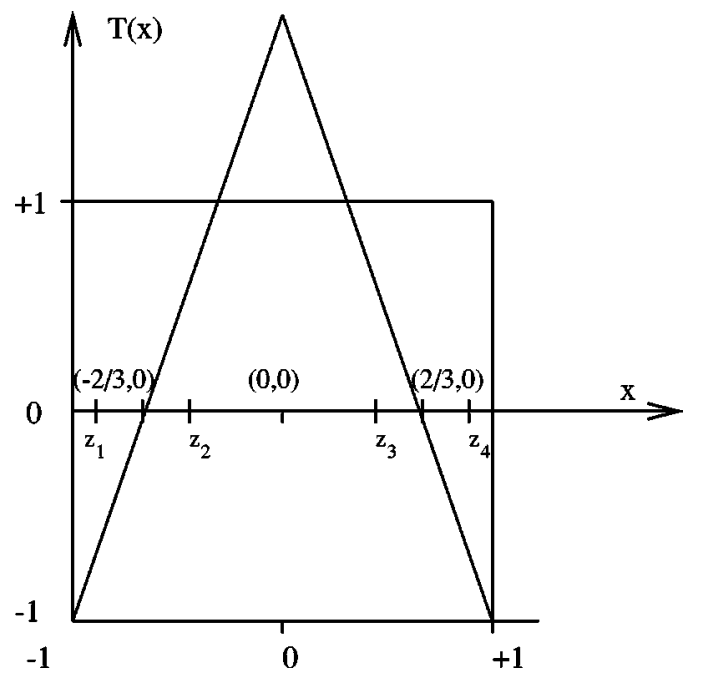

FIG. 2. Graph of the tent map $T(x)=m(1-|x|)-1$ defined in the interval $[-1,+1]$ for $m=3$. The four points $z_{i}$ designate safety points and also $T\left(z_{i}\right)= \pm 2 / 3$.

is buffeted from safety point to safety point without being able to choose between these points (as it is shown in Fig. 3.) There is typically only one that can be reached with $\left|r_{n+1}\right|$ $\leqslant r_{0}$ for each $n$. In the above example calculation, notice that $T\left(x_{n+1}\right)$ is either $-2 / 3$ if $x_{n+1}$ is $z_{4}$ or $+2 / 3$ if $z_{3}$. The protagonist cannot choose whether $T\left(x_{n+1}\right)$ is to be positive or negative (unless $u_{n+1}$ was 0 so that $z_{3}$ and $z_{4}$ were equally close).

The general strategy (called $R$ ) for choosing $r_{n+1}$ is to identify a collection of safety points and choose $x_{0}$ to be one of them and from then on choose $r_{n+1}$ so that $x_{n+1}$ is a safety point. In the case where $2 / 3 \leqslant r_{0}$ and $u_{0} \leqslant r_{0}+2 / 3$, $(k=1)$, there are two safety points, namely, $z_{1}=-2 / 3$ and $z_{2}=2 / 3$. Then if $x_{n}$ is a safety point, $T\left(x_{n}\right)=0$, and the point $T\left(x_{n}\right)+u_{n+1}$ must be in the interval $\left[-u_{0}, u_{0}\right]$. Since $u_{0}$ $\leqslant r_{0}+2 / 3$, each point of the interval is within $r_{0}$ of a safety point. Hence the strategy can be carried out.

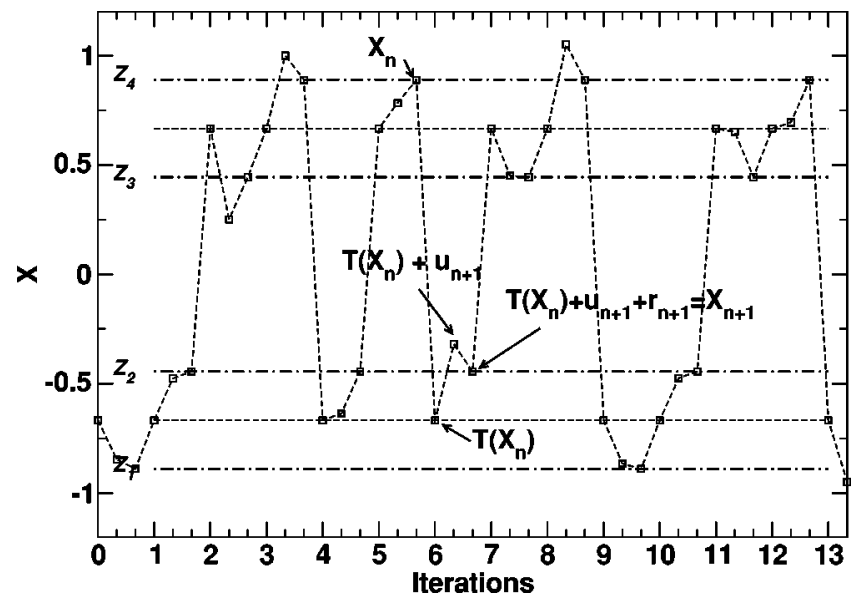

FIG. 3. Evolution of an orbit for $k=2, m=3, r_{0}=2 / 9$, and $u_{0}$ $=4 / 9$. The four dotted-dashed lines represent the safety points $z_{i}$ and the dashed lines represent their images $T\left(z_{i}\right)= \pm 2 / 3$. The points that do not lie over any of these lines represent the steps of the orbit after the influence of the noise $u_{n}$.
In the general case where $2 / 3^{k} \leqslant r_{0}$ and $u_{0} \leqslant r_{0}+2 / 3^{k}$, there are $2^{k}$ safety points, namely, $T^{-k}(0)$ which consists of

$$
\pm 2 / 3^{1} \pm 2 / 3^{2} \pm \cdots \pm 2 / 3^{k} \text { for } k \geqslant 1
$$

Note that $T\left( \pm 2 / 3^{1} \pm 2 / 3^{2} \pm \cdots \pm 2 / 3^{k}\right)$ is a point of the form $\pm 2 / 3^{1} \pm 2 / 3^{2} \pm \ldots \pm 2 / 3^{k-1}$ (which is the single point 0 if $k=1$ ). The argument showing that the strategy can be implemented proceeds as in the special cases discussed above.

We now argue that a guaranteed strategy exists only for the above cases. Hence if $k$ is chosen so that $2 / 3^{k} \leqslant r_{0}$ $<2 / 3^{k-1}$ for some $k \geqslant 1$, and $u_{0}=r_{0}+2 / 3^{k}+\delta$ where $\delta$ $>0$, then no guaranteed strategy exists; in other words, there is a strategy $U$ for choosing the points $u_{n}$ so that the protagonist loses.

Let $S_{k}$ be the set of safe points. The strategy $U$ is to choose $u_{n}$ so that $T\left(x_{n-1}\right)+u_{n}$ is as far as possible. Let $Y_{k}$ be the set $\left\{x:|x-y| \leqslant r_{0}\right.$ for some $y$ in $\left.S_{k}\right\}$. Hence $Y_{k}$ is the set of points that are no more than $r_{0}$ from some safe points. For any point $x_{0}$, there is a $u_{1}$ with $\left|u_{1}\right| \leqslant u_{0}$ such that $T\left(x_{0}\right)+u_{1}$ is not in $Y_{k}$. Hence $x_{1}=T\left(x_{0}\right)+u_{1}+r_{1}$ (with $\left.\left|r_{1}\right| \leqslant r_{0}\right)$ is not a safe point. Let $J_{k}$ be the smallest interval containing $S_{k}$.

If $x_{n}$ is not in $J_{k}$, it is easy to check that strategy $U$ results in $x_{n+1}$ also outside $J_{k}$, but further from $S_{k}$. If $x_{n}$ is in $J_{k}$, let $J^{\prime}$ denote the smallest interval containing $x_{n}$ whose ends are safe points. Strategy $U$ results in $x_{n+1}$ which is in $T\left(J^{\prime}\right)$, which has no points of $S_{k-1}$ in its interior and $x_{n+1}$ is further from $S_{k}$. Furthermore, the length of $T\left(J^{\prime}\right)$ is greater than that of $J^{\prime}$. As the process evolves, the trajectory eventually is outside $J_{k}$, a case which is discussed above.

We have carried out several computer experiments to clarify the applicability of our results. A uniform distributed noise with zero mean value has been used as $u_{n}$, since its only requisite is to be bounded. Obviously, the same results would have been obtained for any other kind of bounded noise. Note that, for this reason, Gaussian noise does not guarantee the survival of the protagonist. For very different values of $k, m$, maximum response $r_{0}$, and maximum perturbation $u_{0}$, being $r_{0} \leqslant u_{0}$, we have iterated the game up to several million steps. As our theorem asserts, the protagonist survives inside the safe region $[-1,1]$ if and only if $u_{0}$ $\leqslant 2 r_{0}$.

An interesting property of the system appears when we analyze the root mean square (RMS) of the control $r_{n}$, which is expressed as

$$
\mathrm{RMS}=\sqrt{\frac{\sum r_{n}^{2}}{n}} .
$$

Figure 4 shows the evolution of the RMS of control when the maximum noise to maximum control ratio is varied, both computationally calculated and analytically derived. We have fixed the control to $r_{0}=2 / 9$ and $u_{0}$ is varied from 0 to $u_{0}$ $=2 r_{0}=4 / 9$. For $u_{0}=0$, that is, in the absence of noise, the control strategy is to push repeatedly the system back to a safety point after the action of the dynamics. The strength of 


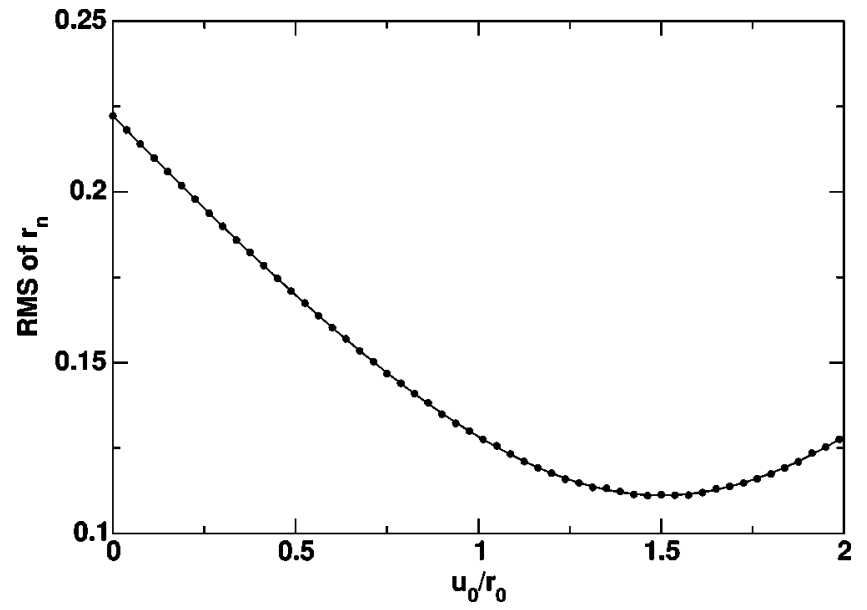

FIG. 4. The control needed decreases in the presence of (weak) noise. The picture shows the root mean square of applied control for different noise-control ratios when $m=3, r_{0}=2 / 9$. The dots were calculated numerically, while the straight line represents the analytical curve.

control is thus constant and equal to the distance to go from the image of a safety point back to any of the safety points. Calling such a distance $d_{k}$, we have $d_{k}=\max _{j}\left\{\min _{i}\left\{\mid z_{i}\right.\right.$ $\left.\left.-T\left(z_{j}\right) \mid\right\}\right\}$, where $z_{i}$ and $z_{j}$ are the safety points, and RMS $=d_{k}$. When noise is switched on, the RMS of control decreases, since in this case the orbit is pushed by the noise $u_{n}$ from the image of a safety point towards one of the $2^{k}$ safety points. This result is in contrast with standard algorithms of chaos control, which aim at stabilizing unstable orbits instead of preimages of the escaping region. For these techniques, a stronger control is needed if noise increases. Finally, for high values of $u_{0} / r_{0}$, the RMS of control shows a minimum and starts to increase again, as there is a value of the noise for which on average the noise places the orbit optimally close to one of the safety points.

The analytical derivation of the curve for RMS is as follows. Looking at Fig. 2, and noticing that the positions of the safety points $z_{i}$ are symmetric, the control needed after a noise displacement $u \leqslant u_{0}$ can be simply written as

$$
|r(u)|=|| u\left|-d_{k}\right|
$$

Indicating with $\sqrt{\left\langle r^{2}\right\rangle}$ the RMS, with $r(u)$ the control needed after a noise displacement $u$, and with $f(u)$ the noise distribution, we obtain the following:

$$
\left\langle r^{2}\right\rangle=\int_{-u_{0}}^{u_{0}} r(u)^{2} f(u) d u=\int_{-u_{0}}^{u_{0}}\left(|u|-d_{k}\right)^{2} f(u) d u
$$

Expanding the expression and distributing the integral, we have

$$
\begin{aligned}
\left\langle r^{2}\right\rangle & =d_{k}^{2}+\int_{-u_{0}}^{u_{0}} u^{2} f(u) d u-2 d_{k} \int_{-u_{0}}^{u_{0}}|u| f(u) d u \\
& =d_{k}^{2}+\left\langle u^{2}\right\rangle-2 d_{k}\langle|u|\rangle
\end{aligned}
$$

To give an example, we can evaluate this expression for the case of uniform noise, that is,

$$
f(u)=\left\{\begin{array}{cc}
\frac{1}{2 u_{0}}, & -u_{0}<u<u_{0} \\
0, & \text { otherwise. }
\end{array}\right.
$$

A straightforward calculation gives

$$
\left\langle u^{2}\right\rangle=\frac{1}{2 u_{0}} \int_{-u_{0}}^{u_{0}} u^{2} d u=\frac{1}{3} u_{0}^{2}
$$

and

$$
\langle|u|\rangle=\frac{1}{2 u_{0}} \int_{-u_{0}}^{u_{0}}|u| d u=\frac{1}{2} u_{0} .
$$

Finally, we obtain that the RMS of control for such distribution is

$$
\sqrt{\left\langle r^{2}\right\rangle}=\sqrt{d_{k}^{2}+\frac{1}{3} u_{0}^{2}-d_{k} u_{0}}
$$

If maximum control $r_{0}$ is set to $d_{k}$, this function has a minimum when $u_{0} / r_{0}=3 / 2$. Figure 4 confirms this result.

The results of this work can be generalized to any unimodal one-dimensional map with a chaotic saddle associated (i.e., with escapes), showing that it is always possible to survive with less control than noise. The relation $u_{0} / r_{0}$, as well as the structure of safety points, will depend on the properties of each map, its symmetry or asymmetry, etc. In order to point this fact, we have developed a similar analytical study for the asymmetric tent map, and the same strategy yields a noise to control ratio of $u_{0} / r_{0}=1+(\mathrm{m} / \mathrm{l})^{k}$, where $m<l$ are the left and right slopes, respectively. It is easy to see that this ratio has a maximum equal to 2 for the symmetric case $m=l$ and a minimum equal to 1 when the right slope is infinitely larger than the left one.

In summary, in this paper we are describing an idea which potentially can be applied to a wide variety of maps with a chaotic saddle, embedded in noisy environments, for an appropriate choice of $r_{0}$ and $u_{0}$. Such an analysis could be far more complex than for the symmetric and asymmetric tent maps, for which the problem can be fully explained analytically. Unlike traditional control theory that tries to steer the state of a system to a precise state, there are situations in which we only have influence in a chaotic environment. The difference between influence and control is roughly speaking $r_{0}<u_{0}$ vs $r_{0}>u_{0}$.

Finally, the information that is needed in order to apply our method is just the approximate position of the safety points. This information might be obtained from time series analysis, suggesting the applicability of this control to real systems. 
We thank James A. Yorke for suggesting to us this problem. J.A. and M.S. acknowledge financial support from MCyT (Spain) under Project No. BFM2000-0967, and from the Universidad Rey Juan Carlos under Project Nos. URJC-
PGRAL-2001/02, URJC-PIGE-02-04, and URJC-GCO2003-16. F. d'O acknowledges financial support from MCyT (Spain) and FEDER, Project No. REN2001-0802-C02-01/ MAR (IMAGEN).
[1] T. Tel, in Directions in Chaos, edited by Hao Bai-Lin, Experimental Study and Characterization of Chaos Vol. 3, (World Scientific, Singapore, 1990), pp. 149-211.

[2] S. Bleher, C. Grebogi, and E. Ott, Physica D 46, 87 (1990).

[3] C. Jung, T. Tel, and E. Zemniak, Chaos 3, 555 (1993).

[4] M. Dhamala and Y.-C. Lai, Phys. Rev. E 59, 1646 (1999).

[5] K. McCann and P. Todzis, Am. Nat. 144, 873 (1994).

[6] I. Dobson and H.-D. Chiang, Syst. Control Lett. 13, 253 (1989).

[7] E. Ott, C. Grebogi, and J.A. Yorke, Phys. Rev. Lett. 64, 1196 (1990)

[8] L. Poon and C. Grebogi, Phys. Rev. Lett. 75, 4023 (1995).

[9] T. Tel, J. Phys. A 24, L1359 (1991).
[10] I.B. Schwartz and I. Triandaf, Phys. Rev. Lett. 77, 4740 (1996).

[11] I. Triandaf and I.B. Schwartz, Phys. Rev. E 62, 3529 (2000).

[12] I.B. Schwartz, I. Triandaf, R. Meucci, and T.W. Carr, Phys. Rev. E 66, 026213 (2002).

[13] E. Akiyama and K. Kaneko, in Advances in Artificial Life, Proceedings of the Fifth European Conference, ECAL99, Lausanne, September 1999, edited by D. Floreano, J.-D. Nicoud, and F. Mondada, Lectures Notes in Computer Science, Vol. 1674, (Springer, Berlin, 1999), pp. 550-565.

[14] E. Akiyama and K. Kaneko, Physica D 147, 221 (2000).

[15] E. Akiyama and K. Kaneko, Physica D 167, 36 (2002). 